\title{
EVALUACIÓN DE DIETAS MERÍDICAS PARA LA CRÍA EN LABORATORIO DE Spodoptera frugiperda (J.E. SMITH) (LEPIDOPTERA: NOCTUIDAE)
}

\section{EVALUATION OF MERIDIC DIETS FOR REARING Spodoptera frugiperda (J.E. SMITH) (LEPIDOPTERA: NOCTUIDAE) UNDER LABORATORIO CONDITIONS}

\author{
Helber Arévalo Maldonado ${ }^{1}$ \\ Ingeborg Zenner de Polanía²
}

\begin{abstract}
RESUMEN
Spodoptera frugiperda es uno de los insectos omnívoros de mayor importancia en el neotrópico americano. Ataca una amplia variedad de cultivos, entre los cuales, se destacan el algodonero y el maíz. De ambas especies existen cultivares transgénicos propuestos como herramientas en su control. Para evaluar su eficacia y monitorear la posible resistencia de insectos a través del tiempo a las toxinas incorporadas, se deben retomar las investigaciones de técnicas de cría con dietas merídicas, a las que se pueden incorporar toxinas del Bacillus thuringiensis y que, además, proporcionan las condiciones nutricionales requeridas y reducen los costos. Esta investigación buscó definir una dieta artificial adecuada para la cría del cogollero del maíz, útil para monitorear su posible resistencia a cultivares transgénicos. La población base, se colectó sobre maíz en el Municipio de Anolaima, Cundinamarca y su desarrollo, se llevó durante tres generaciones consecutivas, sobre las dietas ICRISAT Diet 3, Greene modificada y Shorey \& Hale modificada y alimento natural, hojas de maíz. Con todas las dietas, se logró el desarrollo, pero se presentaron diferencias en la duración del ciclo, en la
\end{abstract}

${ }^{1}$ Ingeniero Agrónomo, Facultad de Ingeniería Agronómica, Universidad de Ciencias Aplicadas y Ambientales U.D.C.A Calle 222No. 54-37, Bogotá D.C. jelber2000@gmail. com

2 I.A. Ph.D. Facultad de Ingeniería Agronómica, Universidad de Ciencias Aplicadas y Ambientales U.D.C.A izenner@ udca.edu.co supervivencia y en el peso de larvas y pupas. La dieta que presentó los mejores resultados fue la dieta ICRISAT, al proporcionar a las larvas una menor duración de desarrollo, un menor número de instares, los mayores pesos de larvas y de pupas, y una supervivencia cercana al $100 \%$. Por esta razón, se sugiere el empleo de la dieta ICRISAT en estudios toxicológicos, donde se requiera la incorporación de las toxinas Cry.

Palabras clave: Cogollero del maíz, técnicas de cría, bioensayos, toxinas Cry, supervivencia.

\section{SUMMARY}

Spodoptera frugiperda is one of the omnivorous insects of mayor importance in the American neo-tropic, due to its attacks of many crops, specially cotton and corn. For both crops, transgenic cultivars are proposed as a tool of pest management. In order to determine the efficacy of $\mathrm{Bt}$ crops and to monitor possible insect resistance through time to the incorporated toxins, research regarding insect rearing on meridic diets, in which Bacillus thuringiensis toxins can be incorporated and furthermore provide the required nutritional conditions and reduce costs, has to be evaluated. This study sought to define an adequate artificial diet for rearing the fall armyworm, which meets the above mentioned characteristics. An initial S. frugiperda population was collected at Anolaima (Department of Cundinamarca) and its development was studied, through three consecutive generations, on ICRISAT Diet 3, modified Greene, modified Shorey $\&$ Hale diet and corn leaves as natural food. With all diets the insect's development was 
attained, but differences in life cycle duration, survival and larval and pupal weight were observed. Best results were obtained with the ICRISAT diet: larvae presented a shorter developmental time, a minor number of instars, and higher weights, for larvae and pupae. Its survival was nearly $100 \%$. For this reason, the use of ICRISAT diet for toxicological studies, where the incorporation of a Cry toxin is required, is suggested.

Key words: Fall armyworm, rearing techniques, bioassays, Cry toxins, survival.

\section{INTRODUCCIÓN}

El gusano cogollero del maíz, Spodoptera frugiperda (J.E. Smith) (Lepidoptera: Noctuidae) es una de las principales plagas del maíz, sorgo, algodonero y arroz en las zonas cálidas y templadas de Colombia (Álvarez E Sánchez 1983). Gallego, en 1946, reporta a esta plaga en el algodonero, cuando aun era conocida como Laphygma frugiperda (Smith E Abbot, 1797) y menciona que, en 1914 y 1915, algunos agricultores la observaron afectando el cultivo de maíz, en forma devastadora, en Antioquia. De la misma forma, Álvarez (1991) en una reseña histórica de la plaga, se refiere a los primeros ataques reportados en el algodonero en el Valle del Cauca, en el año 1962, causando pérdidas económicas, no cuantificadas. El mismo autor menciona que se encuentra en todos los pisos térmicos tropicales y subtropicales como plaga de importancia, desde los 0 a los 2000msnm y desde el norte de Argentina y Chile hasta el sur de Estados Unidos.

El alto costo socio-cultural del control y la dificultad para la obtención de cultivares resistentes incentivaron la búsqueda de alternativas eficientes, económicamente viables y ecológicamente correctas, como las descritas por Cruz et al. (1995) y Grützmacher et al. (2000). Kogan (1998) manifiesta que para que cualquier manejo, sea plenamente utilizable, es necesario conocer la biología de la plaga y, de esta forma entender cómo mantenerla en condiciones de laboratorio, para evaluar diferentes técnicas de control.

Una de las herramientas actualmente utilizadas dentro del manejo integrado de plagas (MIP) en Colombia son las plantas transgénicas, como el algodonero Bollgard ${ }^{\circledR}$ y Bollgard II ${ }^{\circledR}$ o el maíz Yieldgard ${ }^{\circledR}$, que expresan $\mathcal{E}$ endotoxinas del Bacillus thuringiensis (Bt) para el control de algunas plagas de lepidóptera; sin embargo, estas herramientas, se deben monitorear y evaluar, para conocer su eficacia y la resistencia de las plagas objetivo, a través del tiempo (Zenner de Polanía et al. 2008). Una forma de evaluar y de monitorear la resistencia de estos cultivares y su relación con las distintas plagas es el empleo de las dietas merídicas, al permitir el desarrollo del insecto y la incorporación de las $\mathcal{E}$-endotoxinas, a distintas dosis.

Las dietas artificiales proveen un alimento fácil de manejar, eliminan el problema del cultivo de la planta huésped (Etzel \& Legner, 1999 citado por Murúa et al. 2003), pueden disminuir los riesgos de contaminación (Murúa et al. 2003) y brindan los mismos nutrientes durante todo el desarrollo larval de la plaga. Además, permiten mantener constante la mayoría de variables, tales como temperatura, cantidad de alimento, humedad relativa, entre otras, lo cual, es ventajoso para el desarrollo de los respectivos bioensayos.

Entre las dietas más empleadas para la cría del cogollero está la propuesta por Shorey \& Hale (1965), sugerida originalmente para la cría de Trichoplusia ni (Hübner) (Lepidoptera: Noctuidae) y otras especies de nóctuidos, cuya fuente de proteína es el frijol común (Phaseolus vulgaris). Esta dieta ha sido objeto de varios estudios y modificaciones. Bowling (1967) por ejemplo, cambió las relaciones de los ingredientes, mientras que Villacorta $\varepsilon$ Cobo (1978) modificaron la forma de prepararla. Nalim (1991), citado por Souza et al. (2001), la empleo, incluyendo levadura de cerveza y aumentando la concentración de formaldehído, con éxito para la cría de S. frugiperda. Esta dieta, comúnmente denominada de "fríjol", fue usada en el año 2006 por el grupo de investigación "Fitosanidad" de Colombia, para la cría del $S$. frugiperda y para la determinación de concentraciones letales, incorporando dosis seriadas de la toxina Cry1Ac del Bt. Sin embargo, se detectaron problemas de contaminación, básicamente por la bacteria "rosada" Serratia y los hongos Aspergillus y Penicilium, y una alta mortalidad de neonatos, lo que obligó a evaluar otras dietas, objetivo general de este estudio. Para que una dieta pueda ser usada en este tipo de bioensayos de susceptibilidad a Bt, Marçon et al. (2000) establecieron para Ostrinia nubilalis (Hübner), (Lepidoptera: Crambidae), los siguientes criterios: una mortalidad menor del $15 \%$, al séptimo día de desarrollo larval; que las larvas se encuentren entre el tercer y cuarto instar y que, al décimo día, las larvas pesen más de $0,1 \mathrm{mg}$. 
La presente investigación buscó, por lo tanto, otra dieta más apropiada, basado en la evaluación de parámetros biológicos, como la duración de cada estado y de cada instar en particular, la supervivencia, el ciclo de vida, la fecundidad y los pesos de las larvas y pupas y la longevidad de los adultos. Además, se analizó el costo de la dieta y la contaminación microbiana, que en esta se puede desarrollar.

\section{MATERIALES Y MÉTODOS}

La investigación, se efectuó en el Laboratorio de Biotecnología Agrícola, Facultad de Ingeniería Agronómica, Universidad de Ciencias Aplicadas y Ambientales U.D.C.A. Las condiciones ambientales de temperatura y de humedad relativa fueron $24 \pm 3^{\circ} \mathrm{C}$ y $70 \pm 5 \%$ HR, respectivamente.

Larvas de $S$. frugiperda, consideradas $\mathrm{F}_{0}$, se recolectaron en un lote de maíz híbrido (CIMMYT), en el municipio de Anolaima, Cundinamarca, situado a 04²4'15,3" N y a $74^{\circ} 29^{\prime}$ ' $46,7^{\prime \prime}$ E. Su cría en el laboratorio, se realizó sobre hojas de maíz, cambiadas cada segundo día, hasta la formación de la pupa. En pupa, se determinó el sexo, según But $\mathcal{E}$ Cantu (1962) citado por García et al. (2006) y se dejaron en el mismo recipiente de cría, vaso plástico de capacidad de $50 \mathrm{~mL}$, con tapa inyectada (Envapac Ltda., Bogotá), con una mota de algodón, humedecida con agua destilada. Cuando los adultos emergieron, se corroboró su sexo basado en la coloración de las alas anteriores, como lo sugiere Gallego (1946). Los adultos nacidos el mismo día, se dispusieron por parejas en una cámara de oviposición, para la cual, se adaptaron porrones de vidrio de 4,0L de capacidad (Peldar S.A., Soacha, Cundinamarca), cubierta con una tela tipo tole de color blanco, con tiras de papel de color blanco o beige, para poder observar fácilmente las posturas, colocadas desde el borde hasta el fondo de la cámara. Los adultos fueron alimentados diariamente, con una solución acuosa con $10 \%$ de azúcar y $1 \%$ de acido ascórbico. De la segunda noche de oviposición, se separaron 160 huevos, tomando en cuenta lo mencionado por Garcia et al. (2006), quienes encontraron que las posturas de esta noche son las más viables.

Al eclosionar estas posturas, se tomaron 40 larvas neonatas para cada tratamiento, las cuales, se dispusieron de manera individual, en las cuatro dietas: 1) ICRISAT Diet 3 a base de garbanzo (T2), preparada de acuerdo a la fórmula proporcionada por el doctor $\mathrm{H}$. Sharma, del Instituto Internacional de Investigación en Cultivos para las Zonas Tropicales Semiáridas (ICRISAT) Patancheru, Andhra Pradesh, India; 2) Greene et al. (1976) modificada por Parra (2001) (García et al. 2006) (T3); 3) Shorey \& Hale (1965) modificada por Bowling (1967) y preparada según Villacorta \& Cobo (1978) (T4) (Tabla 1) y, 4) el sustrato natural hojas de maíz (T1). Se emplearon los mismos vasos plásticos de $50 \mathrm{~mL}$ de capacidad con tapa inyectada, arriba mencionados, llenados con $7 \mathrm{~mL}$ de dieta, continuándose el desarrollo de los individuos sobrevivientes utilizando la metodología descrita anteriormente.

Los parámetros biológicos, se determinaron durante tres generaciones consecutivas. La duración de cada instar, de cada estado y del ciclo total de huevo a adulto, incluyendo la supervivencia, se contabilizó diariamente. Con estos datos, se construyó la curva de supervivencia y la tabla de mortalidad. Para comprobar las mudas, se separaron las cápsulas cefálicas una vez ocurría la ecdisis. A los adultos obtenidos, se tomaron los periodos de pre-oviposición, oviposición y pos-oviposición y la longevidad de éstos. La fecundidad, se estableció separando y contando diariamente los huevos colocados. Teniendo en cuenta las capas de huevos, de las cuales, consta cada postura de S. frugiperda, para contabilizar el número de huevos, se halló, inicialmente, el área de cada postura y, luego, se dividió por el área, previamente establecido, que ocupa cada huevo. Con base a los datos de sobrevivencia y oviposición de cada hembra, se construyeron las gráficas de fecundidad bruta $(\mathrm{Mx})$. El peso larval, se tomó al décimo día, aceptando para los bioensayos con toxinas Cry, la recomendación de Marçon et al. (2000), quienes trabajando con $O$. nubilalis consideraron muertas las larvas con un peso menor a 0,1mg. Las pupas, se pesaron a las 24 horas de su formación.

El costo de cada dieta, se determinó basado en el valor comercial de los ingredientes y el consumo de cada larva hasta la formación de la pupa, $1,5 \mathrm{~mL}$ de dieta según Murúa et al. (2003) y la supervivencia.

Las evaluaciones, se establecieron bajo un arreglo de bloques al azar donde las generaciones fueron los bloques y las dietas los tratamientos. Los datos fueron sometidos a un análisis de varianza y de pruebas de Tukey, empleando el programa SAS Institute Inc. 
Tabla 1. Ingredientes utilizados en la preparación de las dietas artificiales (ICRISAT Diet 3, Shorey \& Hale modificada y Greene modificada).

\begin{tabular}{|c|c|c|c|}
\hline $\begin{array}{c}\text { Ingredientes } \\
\text { para preparar 500mL }\end{array}$ & $\begin{array}{c}\text { ICRISAT } \\
\text { Dieta No. 3 }\end{array}$ & $\begin{array}{c}\text { Shorey \& Hale } \\
\text { (modificada) }\end{array}$ & Greene (modificada) \\
\hline Fracción A & & & \\
\hline Agua & $112 \mathrm{~mL}$ & $200 \mathrm{~mL}$ & $200 \mathrm{~mL}$ \\
\hline Fríjol blanco & & & $29,2 \mathrm{~g}$ \\
\hline Frijol calima & & $100 \mathrm{~g}$ & \\
\hline Harina de garbanzo & $75 \mathrm{~g}$ & & $11,75 \mathrm{~g}$ \\
\hline Harina de soja & & & $23,5 \mathrm{~g}$ \\
\hline Germen de trigo & & & $14,75 \mathrm{~g}$ \\
\hline Levadura de cerveza & & & $8,9 \mathrm{~g}$ \\
\hline Levadura & $12 \mathrm{~g}$ & $15 \mathrm{~g}$ & $1,07 \mathrm{~g}$ \\
\hline Leche en polvo & & & $1,4 \mathrm{~g}$ \\
\hline Metil paraben Nipagina & $1,25 \mathrm{~g}$ & $1 \mathrm{~g}$ & $0,7 \mathrm{~g}$ \\
\hline Ácido ascórbico & $1,17 \mathrm{~g}$ & $1,5 \mathrm{~g}$ & 2 tabletas \\
\hline Ácido sórbico & $0,75 \mathrm{~g}$ & $0,5 \mathrm{~g}$ & \\
\hline Tableta multivitamínica & 1 tabletas & & \\
\hline Ambramicina & $0,04 \mathrm{~g}$ & & $1,4 \mathrm{~mL}$ \\
\hline Tetraciclina & & & $5,45 \mathrm{~g}$ \\
\hline Fracción B & & & $202 \mathrm{~mL}$ \\
\hline Formaldehído (38\%) & & $1 \mathrm{~mL}$ & \\
\hline Agar & $4,37 \mathrm{~g}$ & $6 \mathrm{~g}$ & \\
\hline Agua & $200 \mathrm{~mL}$ & $125 \mathrm{~mL}$ & \\
\hline
\end{tabular}

\section{RESULTADOS Y DISCUSIÓN}

Los resultados del tiempo de desarrollo de los diferentes estados de $S$. frugiperda, obtenidos en cada una de las dietas y en el alimento natural, durante las tres generaciones, se consignaron en la tabla 2. Se observa que estos parámetros presentaron diferencias en el tiempo de desarrollo del estado de larva y del ciclo total de vida. Los menores tiempos en estado de larva y ciclo, sin importar la generación, se obtuvieron con la dieta ICRISAT y el alimento natural. Estos resultados, confirman la variación en la duración larval, la cual, se puede deber a la calidad y al tipo de alimento, encontrado con anterioridad por Álvarez E Sánchez (1983) y por Murúa et al. (2003). Es importante fijarse que la duración del estado larval varía de 13 a 37 días. El tiempo que el insecto pasa en el estado larval es variable. Etcheverry (1957) citado por Sarmento et al. (2002), obtuvieron una duración, bajo las mismas condiciones ambientales, entre los 12 a 24 días, mientras que Murúa et al. (2003) observaron una duración similar, con la dieta cuya fuente proteica es el frijol blanco y germen de trigo, al tener un tiempo promedio de 25,85 $\pm 0,53$ días. Todos las dietas ofrecidas en esta evaluación mostraron una menor duración, al compararlos con dietas empleadas por Murúa et al. (2003), a base de maíz colorado y germen de trigo, transcurrió de 37,4 46,3 días y, a base de arroz, fécula de maíz y germen de trigo, duración larval de $43,5 \pm 3,37$ días, indicando, claramente, la influencia de la dieta alimenticia.

También es pertinente mencionar que esta duración depende de las características genéticas, que pueden variar por la existencia de diferentes biotipos o razas, como la que ataca a los cultivos de pastos y arroz y la que ataca al algodonero y al maíz (Zenner et al. 2007, Busato et al. 2006). Estos datos, a su vez, corroboran que la larva puede prolongar o acortar el ciclo, dependiendo del alimento que disponga, como se mencionó, en observaciones de campo por Vélez (1979) citado por Vélez (1985), debido a que el cogollero prolonga su ciclo cuando se alimenta con algodón y lo reduce cuando se alimenta con maíz. El ciclo total del insecto estuvo 
Tabla 2. Duración en días de los estados de huevo, larva, pupa, adulto y del ciclo completo del cogollero del maíz, Spodoptera frugiperda.

\begin{tabular}{|c|c|c|c|c|c|c|}
\hline \multirow[t]{2}{*}{ Generaciones } & \multirow[t]{2}{*}{ Tratamientos } & \multicolumn{5}{|c|}{$\begin{array}{l}\text { Estado de desarrollo } \\
\text { Promedio (Días) } \pm \text { DS. }\end{array}$} \\
\hline & & Huevo & Larva & Pupa & Adulto & Ciclo \\
\hline \multirow{4}{*}{ F1 } & $\mathrm{T} 1$ & $\begin{array}{c}3 \pm 0 \mathrm{~A} \\
(3)[40]\end{array}$ & $\begin{array}{c}22,29 \pm 2,31 A^{*} \\
(18-27)[24]\end{array}$ & $\begin{array}{c}12,85 \pm 2,08 \mathrm{~A} \\
(10-17)[20]\end{array}$ & $\begin{array}{c}8,66 \pm 2,95 \mathrm{~A} \\
(4-14)[18]\end{array}$ & $\begin{array}{c}46,83 \pm 3,50 \mathrm{AB} \\
(40-51)[18]\end{array}$ \\
\hline & T2 & $\begin{array}{l}3 \pm 0 \mathrm{~A} \\
(3)[40]\end{array}$ & $\begin{array}{c}19,55 \pm 1,17 A^{*} \\
(19-22)[38]\end{array}$ & $\begin{array}{c}14,68 \pm 0,96 \mathrm{~A} \\
(13-17)[38]\end{array}$ & $\begin{array}{c}7,97 \pm 3,39 A \\
(0-13)[38]\end{array}$ & $\begin{array}{c}45,21 \pm 3,62 A \\
(36-51)[38]\end{array}$ \\
\hline & T3 & $\begin{array}{l}3 \pm 0 \mathrm{~A} \\
(3)[40]\end{array}$ & $\begin{array}{c}23,27 \pm 2,57 \mathrm{~B}^{*} \\
(15-28)[37]\end{array}$ & $\begin{array}{c}10,94 \pm 1,17 \mathrm{~A} \\
(9-14)[37]\end{array}$ & $\begin{array}{c}9,24 \pm 4,42 \mathrm{~A} \\
(0-19)[37]\end{array}$ & $\begin{array}{c}46,45 \pm 5,29 B C \\
(34-57)[37]\end{array}$ \\
\hline & T4 & $\begin{array}{c}3 \pm 0 \mathrm{~A} \\
(3)[40]\end{array}$ & $\begin{array}{c}30,06 \pm 4,62 B^{*} \\
(25-40)[15]\end{array}$ & $\begin{array}{c}12,57 \pm 0,78 \mathrm{~A} \\
(11-13)[7]\end{array}$ & $\begin{array}{c}6,42 \pm 3,73 \mathrm{~A} \\
(1-11)[7]\end{array}$ & $\begin{array}{c}48,71 \pm 5,05 C \\
(41-54)[7]\end{array}$ \\
\hline \multirow{4}{*}{ F2 } & $\mathrm{T} 1$ & $\begin{array}{c}3 \pm 0 \mathrm{~A} \\
(3)[40]\end{array}$ & $\begin{array}{c}18,87 \pm 2,17 A^{*} \\
(16-24)[31]\end{array}$ & $\begin{array}{c}10,88 \pm 1,21 \mathrm{~A} \\
(8-13)[27]\end{array}$ & $\begin{array}{c}9,59 \pm 7,30 A \\
(0-32)[27]\end{array}$ & $\begin{array}{c}42,03 \pm 8,09 A B \\
(32-64)[27]\end{array}$ \\
\hline & T2 & $\begin{array}{c}3 \pm 0 \mathrm{~A} \\
(3)[40]\end{array}$ & $\begin{array}{c}18,97 \pm 2,32 A^{*} \\
(16-26)[40]\end{array}$ & $\begin{array}{l}11,75 \pm 1,12 \mathrm{~A} \\
(10-15)[40]\end{array}$ & $\begin{array}{l}9,5 \pm 4,39 \mathrm{~A} \\
(0-16)[40]\end{array}$ & $\begin{array}{l}43,22 \pm 5,76^{\mathrm{a}} \\
(30-53)[40]\end{array}$ \\
\hline & T3 & $\begin{array}{l}3 \pm 0 \mathrm{~A} \\
(3)[40]\end{array}$ & $\begin{array}{c}26,70 \pm 2,40 \mathrm{~B}^{*} \\
(19-32)[37]\end{array}$ & $\begin{array}{l}11,79 \pm 0,76 \mathrm{~A} \\
(10-13,5)[32]\end{array}$ & $\begin{array}{c}8,51 \pm 3,56 \mathrm{~A} \\
(0-12)[32]\end{array}$ & $\begin{array}{c}49,81 \pm 4,25 \mathrm{BC} \\
(41,56)[32]\end{array}$ \\
\hline & $\mathrm{T} 4$ & $\begin{array}{l}2 \pm 0 \mathrm{~A} \\
\text { (3) }[40]\end{array}$ & $\begin{array}{c}24,3 \pm 2,93 B^{*} \\
(15-27)[20]\end{array}$ & $\begin{array}{l}13,4 \pm 1,77 \mathrm{~A} \\
(10-17)[10]\end{array}$ & $\begin{array}{l}11,4 \pm 1,57 \mathrm{~A} \\
(9-13)[10]\end{array}$ & $\begin{array}{l}51,1 \pm 2,72 C \\
(46-54)[10]\end{array}$ \\
\hline \multirow{4}{*}{ F3 } & $\mathrm{T} 1$ & $\begin{array}{l}4 \pm 0 \mathrm{~A} \\
(3)[40]\end{array}$ & $\begin{array}{l}18,5 \pm 2,81 A^{*} \\
(13-24)[32]\end{array}$ & $\begin{array}{l}11,13 \pm 1,05 \mathrm{~A} \\
(10-13)[29]\end{array}$ & $\begin{array}{c}8,19 \pm 2,63 \mathrm{~A} \\
(2-11)[26]\end{array}$ & $\begin{array}{c}41,84 \pm 3,83 \mathrm{AB} \\
(33-47)[26]\end{array}$ \\
\hline & T2 & $\begin{array}{l}4 \pm 0 \mathrm{~A} \\
(4)[40]\end{array}$ & $\begin{array}{c}17,94 \pm 1,63 A^{*} \\
(16-23)[36]\end{array}$ & $\begin{array}{c}11,23 \pm 1,12 \mathrm{~A} \\
(9-14)[34]\end{array}$ & $\begin{array}{c}9,05 \pm 3,69 \mathrm{~A} \\
(0-15)[34]\end{array}$ & $\begin{array}{c}42,08 \pm 3,71 \mathrm{~A} \\
(32-48)[34]\end{array}$ \\
\hline & T3 & $\begin{array}{c}3 \pm 0 \mathrm{~A} \\
(3)[40]\end{array}$ & $\begin{array}{c}29,76 \pm 3,80 \mathrm{~B}^{*} \\
(20-37)[34]\end{array}$ & $\begin{array}{l}12,19 \pm 0,8 \mathrm{~A} \\
(10-14)[26]\end{array}$ & $\begin{array}{c}7,76 \pm 3,61 \mathrm{~A} \\
(0-11)[26]\end{array}$ & $\begin{array}{c}52,30 \pm 5,61 \mathrm{BC} \\
(41-61)[26]\end{array}$ \\
\hline & $\mathrm{T} 4$ & $\begin{array}{l}4 \pm 0 \mathrm{~A} \\
\text { (3) }[40]\end{array}$ & $\begin{array}{c}22,73 \pm 5,18 \mathrm{~B}^{*} \\
(17-31)[8]\end{array}$ & $\begin{array}{c}12,75 \pm 0,88 \mathrm{~A} \\
(12-14)[8]\end{array}$ & $\begin{array}{l}10 \pm 2,26 \mathrm{~A} \\
(6-13)[8]\end{array}$ & $\begin{array}{c}49,12 \pm 4,18 C \\
(43-55)[8]\end{array}$ \\
\hline
\end{tabular}

T1: Alimento natural (hojas de maíz); T2: Dieta ICRISAT; T3: Dieta Shorey \& Hale modificada; T4: Dieta Greene modificada.

F1: primera generación; F2: segunda generación, F3: tercera generación (de laboratorio).

Los promedios con las mismas letras no presentan diferencias significativas, según prueba de Tukey.

Los valores entre paréntesis expresan el rango y entre los corchetes, el número de unidades experimentales.

${ }^{*}$ Las generaciones presentaron diferencias significativas $\mathrm{P} \leq 0,05$.

determinado por su duración del estado larval, por lo tanto, se considera que la menor duración del ciclo, se dio con la dieta ICRISAT, que corresponde a la dieta de mayor valor nutritivo para la larva.

La duración de larva presentó diferencias significativas entre generaciones $(P=0,02)$, debido a que todos los tratamientos, exceptuando el T3, redujeron su duración. Este fenómeno, se puede explicar por la adaptación paulatina que tiene el insecto a diferentes sustratos alimenticios; en este caso, el tratamiento tres, podría carecer de algún elemento nutricional, que sólo se percibe a medida que avanzan las generaciones.

En el presente trabajo, se comprobó que el número de instares, no solo depende de la temperatura media, sino también de la calidad y del tipo de alimento. Es preciso anotar que no se presentaron más de ochos instares, 
mientras que Murúa et al. (2003), obtuvieron hasta diez en la dieta a base de arroz, fécula de maíz y germen de trigo, donde, además, la duración de este estado, fue más prolongado. Las larvas en este estudio pasaron por seis a ocho instares, sin importar el sustrato alimenticio, aunque en promedio, durante las tres generaciones, el $89 \%$ de las larvas alimentadas con la dieta ICRISAT, presentaron seis instares.

Uno de los datos más importantes para estimar la calidad de dietas es el peso de las larvas, en un momento determinado, y el peso de la pupa, al día siguiente de su formación (Bustillo, 1979). Se asume que un mayor peso, en un momento dado, puede asegurar una mayor supervivencia. Con relación a este parámetro, se observa en la figura 1 , que los pesos larvales al décimo día presentaron diferencias altamente significativas entre tratamientos $(P<0,0001)$ y significativas entre generaciones $(P=0,02)$; los mayores, se evidenciaron en todas las generaciones con la dieta ICRISAT. En todas las generaciones, los menores se observaron con la dieta Greene modificada, pero aún estos pesos fueron mayores a 0,1mg, uno de los parámetros para el uso de las dietas en bioensayos con E-endotoxinas (Marçon et al. 2000).
En las tres generaciones, los mayores pesos de las pupas, a las 24 horas (Figura 1), se registraron con la dieta ICRISAT, pesos que incluso fueron mayores a los encontrados en el alimento natural. Pantoja et al. (1987) encontraron pesos de pupa entre 153,8 \pm 11 y $197,2 \pm 17,5 \mathrm{mg}$, al alimentar las larvas con hojas de arroz. Estos datos son similares a los obtenidos en el tratamiento uno, donde las larvas fueron alimentadas con hojas de maíz, lo cual, sugiere que éste es el peso de una pupa bajo condiciones naturales. La dieta que más se aproxima a estos valores es la del T3, dato muy provechoso, si lo que desea el investigador es tener una dieta que le brinde un peso similar al encontrado en el ambiente natural de la especie. Villacorta \& Cobo (1978), encontraron pesos similares, $195 \mathrm{mg}$, al alimentar las larvas con esta dieta. Los pesos encontrados en el tratamiento cuatro fueron menores a los reportados por Busato et al. (2006), al alimentar las larvas con la misma dieta, ya que variaron entre $254 \pm 2$ y $272 \pm 2 \mathrm{mg}$.

Contrario a lo esperado, los periodos de oviposición (Tabla 3) no presentaron diferencias significativas, lo cual, muestra que el alimento de la fase larval no tuvo mayor efecto sobre su duración. Estos datos concuerdan

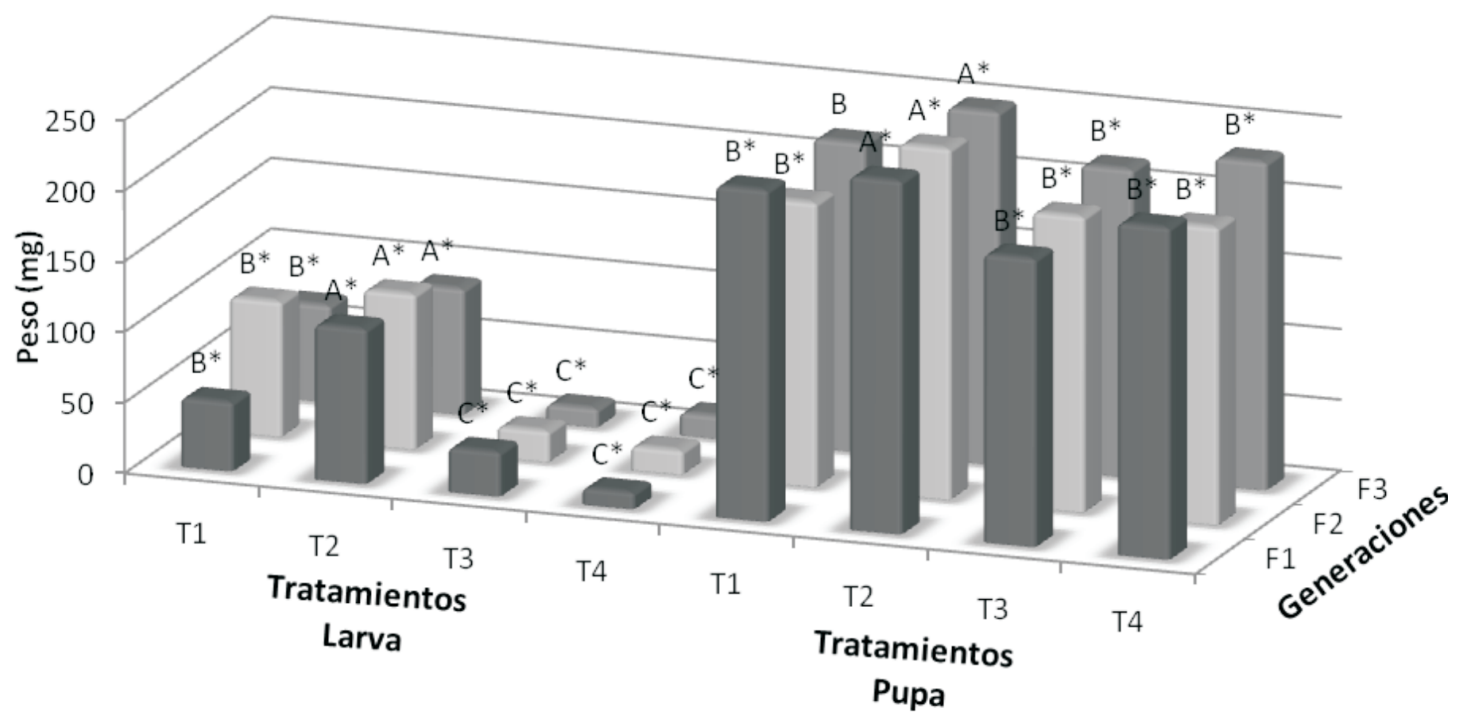

Figura 1. Peso de larvas Spodoptera frugiperda al décimo día y de pupas 24 horas después de su formación, en tres dietas merídicas y hojas de maíz, durante tres generaciones.

T1: Alimento natural (hojas de maíz); T2: Dieta ICRISAT; T3: Dieta Shorey \& Hale modificada; T4: Dieta Greene modificada.

F1: primera generación; F2: segunda generación, F3: tercera generación (en laboratorio).

Los promedios con las mismas letras no presentan diferencias significativas, según prueba de Tukey

* Las generaciones presentaron diferencias significativas $P \leq 0,05$. 
con los obtenidos por Smith (1921). En la tabla 3, también se observa la producción media de huevos por hembra, que tampoco arrojó diferencias significativas entre las tres dietas y el alimento natural; sin embargo, en todos los casos, la producción media de huevos fue menor a la encontrada por Busato et al. (2006), quienes contabilizaron 1565 huevos.

La tasa máxima de fecundidad bruta (Figura 2), se registró entre los 36 a 47 días. Exceptuando las hembras del T4 en la tercera generación, se cumplieron con las características: cópula normal, huevos viables en más del $75 \%$ y progenie fértil. La ausencia de posturas de las hembras T4, se debió a la ausencia de cópula, por no coincidir los machos con las hembras y el bajo número de sobrevivientes hasta el estado adulto. Busato et al. (2004), encontraron resultados similares, con una tasa máxima de fecundidad, el día 35, para una población procedente de maíz, el día 36, para una población de maíz alimentada con arroz y, de nuevo, el día 35 para una población de arroz alimentada con maíz. Es importante observar que la población evaluada en esta investigación es más fecunda que la población evaluada por Busato et al. (2004), al producir más de 160 individuos en un día.

Tabla 3. Periodos de oviposición y fecundidad de Spodoptera frugiperda en tres dietas artificiales y alimento natural (hojas de maíz), durante tres generaciones consecutivas.

\begin{tabular}{|c|c|c|c|c|c|}
\hline Generaciones & Tratamientos & $\begin{array}{l}\text { PRE } \\
\text { (días) }\end{array}$ & $\begin{array}{l}\text { OVIPOSICIÓN } \\
\text { (días) }\end{array}$ & $\begin{array}{l}\text { POS } \\
\text { (días) }\end{array}$ & $\begin{array}{c}\text { FECUNDIDAD } \\
\text { TOTAL }\end{array}$ \\
\hline \multirow{4}{*}{ F1 } & $\mathrm{T} 1$ & $\begin{array}{c}2,75 \pm 1,03 \mathrm{~A} \\
(2-4)[8]\end{array}$ & $\begin{array}{c}3,75 \pm 2,25 \mathrm{~A} \\
(1-7)[8] \\
\end{array}$ & $\begin{array}{c}2,5 \pm 2,56 \mathrm{~A} \\
(0-7)[8] \\
\end{array}$ & $\begin{array}{c}742,9 \pm 639,8 \mathrm{~A} \\
(0-1810)[10]\end{array}$ \\
\hline & $\mathrm{T} 2$ & $\begin{array}{c}2,63 \pm 0,8 \mathrm{~A} \\
(2-4)[11]\end{array}$ & $\begin{array}{c}4,63 \pm 1,5 \mathrm{~A} \\
(2-7)[11]\end{array}$ & $\begin{array}{c}1,54 \pm 1,63 \mathrm{~A} \\
(0-5)[11]\end{array}$ & $\begin{array}{l}884,4 \pm 422,3 \mathrm{~A} \\
(23-1587)[11]\end{array}$ \\
\hline & T3 & $\begin{array}{l}3 \pm 0,67 \mathrm{~A} \\
(2-4)[14] \\
\end{array}$ & $\begin{array}{l}5 \pm 1,88 \mathrm{~A} \\
(2-9)[14] \\
\end{array}$ & $\begin{array}{c}3 \pm 2^{\mathrm{a}} \\
(0-6)[14]\end{array}$ & $\begin{array}{c}905,8 \pm 623,7 \mathrm{~A} \\
(0-2305)[16] \\
\end{array}$ \\
\hline & T4 & $\begin{array}{c}3 \pm 1 A \\
(2-4)[3]\end{array}$ & $\begin{array}{c}4 \pm 1,1,73 \mathrm{~A} \\
(3-6)[3]\end{array}$ & $\begin{array}{c}2,66 \pm 0,57 \mathrm{~A} \\
(2-3)[3]\end{array}$ & $\begin{array}{c}672,6 \pm 501 \mathrm{~A} \\
(174-1176)[3]\end{array}$ \\
\hline \multirow{4}{*}{ F2 } & $\mathrm{T} 1$ & $\begin{array}{c}2,37 \pm 0,51 \mathrm{~A} \\
(2-3)[8] \\
\end{array}$ & $\begin{array}{c}6,75 \pm 1,38 \mathrm{~A} \\
(4-9)[8] \\
\end{array}$ & $\begin{array}{c}3,37 \pm 3,37 \mathrm{~A} \\
(0-10)[8]\end{array}$ & $\begin{array}{c}536,3 \pm 664,4 \mathrm{~A} \\
(0-2004)[16] \\
\end{array}$ \\
\hline & $\mathrm{T} 2$ & $\begin{array}{c}2,92 \pm 0,73 \mathrm{~A} \\
(2-4)[14]\end{array}$ & $\begin{array}{c}4,57 \pm 2,27 \mathrm{~A} \\
(2-10)[14]\end{array}$ & $\begin{array}{c}4,42 \pm 2,50 \mathrm{~A} \\
(0-10)[14] \\
\end{array}$ & $\begin{array}{c}1008,9 \pm 687,4 A \\
(0-2306)[15]\end{array}$ \\
\hline & T3 & $\begin{array}{c}2,23 \pm 1,01 \mathrm{~A} \\
(1-4)[13] \\
\end{array}$ & $\begin{array}{c}3,69 \pm 1,49 A \\
(1-5)[13] \\
\end{array}$ & $\begin{array}{c}3,69 \pm 1,49 \mathrm{~A} \\
(1-5)[13]\end{array}$ & $\begin{array}{l}705 \pm 515,9 \mathrm{~A} \\
(0-2024)[15] \\
\end{array}$ \\
\hline & T4 & $\begin{array}{c}2,75 \pm 0,95 \mathrm{~A} \\
(2-4)[4]\end{array}$ & $\begin{array}{c}4 \pm 1,82 \mathrm{~A} \\
(2-6)[4] \\
\end{array}$ & $\begin{array}{c}4,5 \pm 1,29 \mathrm{~A} \\
(3-6)[4]\end{array}$ & $\begin{array}{c}890 \pm 568,8 \mathrm{~A} \\
(320-1636)[4] \\
\end{array}$ \\
\hline \multirow{4}{*}{ F3 } & $\mathrm{T} 1$ & $\begin{array}{c}3,09 \pm 0,83 \mathrm{~A} \\
(2-4)[11] \\
\end{array}$ & $\begin{array}{c}4,36 \pm 1,62 \mathrm{~A} \\
(1-7)[11] \\
\end{array}$ & $\begin{array}{c}1,36 \pm 1,50 \mathrm{~A} \\
(0-4)[11] \\
\end{array}$ & $\begin{array}{c}614,7 \pm 470,2 \mathrm{~A} \\
(0-1407)[14]\end{array}$ \\
\hline & T2 & $\begin{array}{c}2,63 \pm 0,67 \mathrm{~A} \\
(2-4)[11] \\
\end{array}$ & $\begin{array}{c}5,09 \pm 0,7 A \\
(4-6)[11] \\
\end{array}$ & $\begin{array}{c}2,36 \pm 1,56 \mathrm{~A} \\
(0-5)[11] \\
\end{array}$ & $\begin{array}{c}1008,6 \pm 788,4 \mathrm{~A} \\
(0-2294)[15] \\
\end{array}$ \\
\hline & T3 & $\begin{array}{c}2,44 \pm 0,72 \mathrm{~A} \\
(2-4)[9] \\
\end{array}$ & $\begin{array}{l}5 \pm 1,32 A \\
(2-6)[9] \\
\end{array}$ & $\begin{array}{l}1,66 \pm 1^{\mathrm{a}} \\
(0-3)[9]\end{array}$ & $\begin{array}{c}1004,8 \pm 681,8 \mathrm{~A} \\
(0-1950)[11] \\
\end{array}$ \\
\hline & $\mathrm{T} 4$ & N.D. & N.D. & N.D. & N.D. \\
\hline
\end{tabular}

T1: Alimento natural (hojas de maíz); T2: Dieta ICRISAT; T3: Dieta Shorey \& Hale; T4: Dieta Greene.

F1: primera generación; F2: segunda generación, F3: tercera generación (de laboratorio).

Los promedios con las mismas letras no presentan diferencias significativas, según prueba de Tukey.

Los valores entre paréntesis expresan el rango y entre los corchetes, el número de unidades experimentales. 

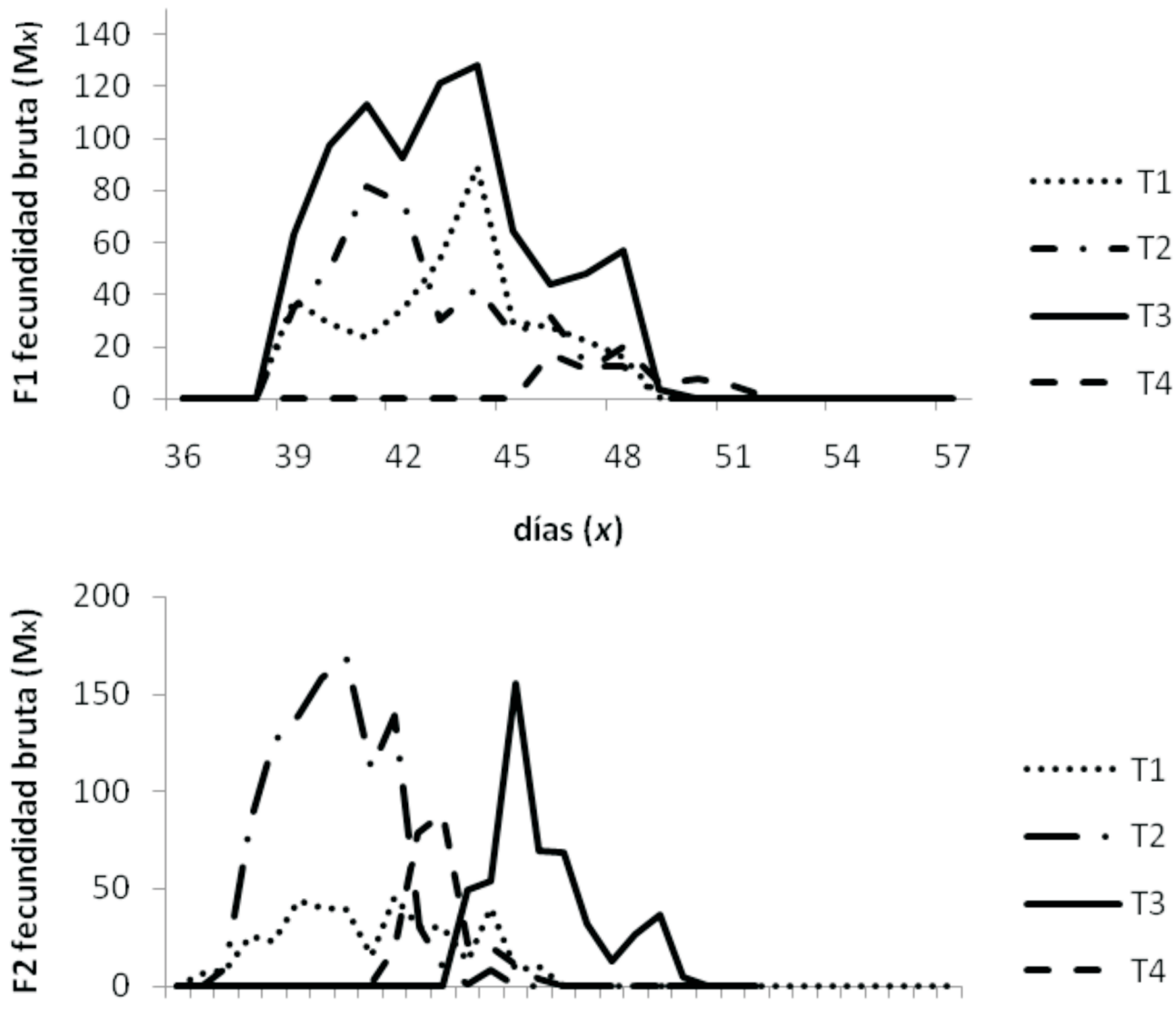

3234363840424446485052545658606264

días $(x)$

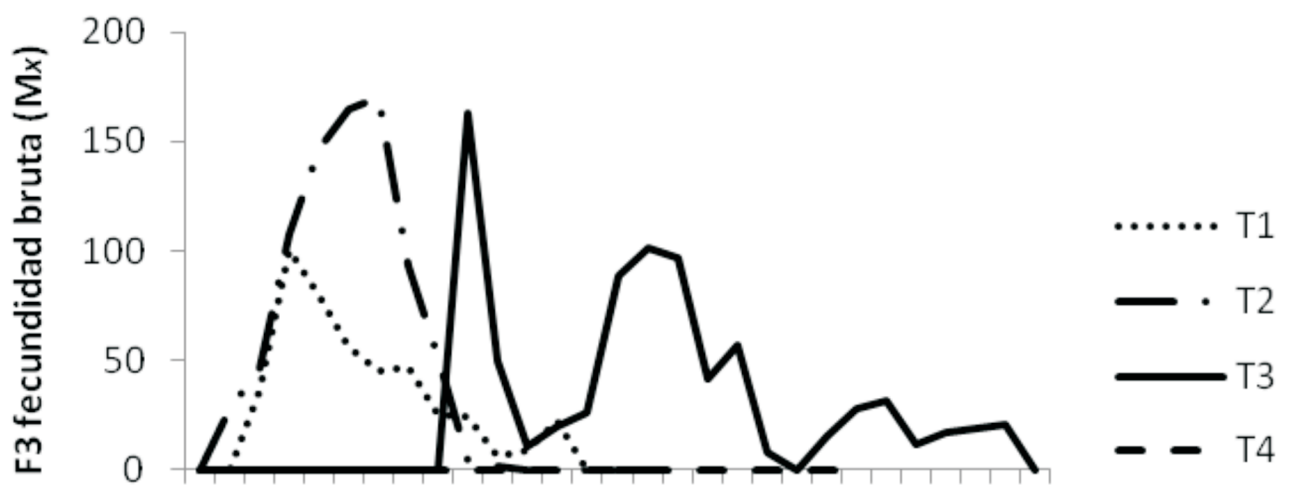

333537394143454749515355575961

días $(x)$

Figura 2. Fecundidad bruta (Mx), para Spodoptera frugiperda, en tres dietas artificiales y alimento natural (hojas de maíz), durante tres generaciones consecutivas.

T1: Alimento natural (Hojas de Maíz); T2: Dieta ICRISAT; T3: Dieta Shorey \& Hale modificada; T4: Dieta Greene modificada.

F1: primera generación; F2: segunda generación, F3: tercera generación (en laboratorio). 
Con relación a las curvas de supervivencia (Figura 3), se observa que los tratamientos dos y tres describen una curva idealizada tipo I, de Deevey (1947) citado por Ravinovich (1980), en la primera generación, lo que indica que las poblaciones tuvieron una probabilidad constante y cercana al $100 \%$ de sobrevivir durante todos los estados de desarrollo, hasta prácticamente el final, ocurriendo la mortalidad en el estado adulto. Este comportamiento es el ideal, si el objetivo es una cría masiva o su empleo en bioensayos y, solo es posible encontrarlo, con el uso de una dieta y bajo condiciones controladas, ya que la dieta permite la regulación en nutrición del insecto y su aislamiento del ambiente natural. Lo anterior, contrario a lo obtenido en los tratamientos uno y cuatro, donde las curvas se aproximan a curvas de tipo II y III, las cuales, indican que la mortalidad se puede producir en cualquier estado de desarrollo o que la mayor mortalidad, se presenta en estados juveniles, respectivamente, algo indeseable. La mortalidad en estos casos ocurrió, en su mayoría, en el estado larval, en los instares próximos a la formación de la pupa y estuvo determinada por dos factores importantes: la contaminación de la dieta, por Penicilium, causando un efecto anti-alimentario y la no adaptación del insecto a los diferentes sustratos.

En cuanto a los ingredientes, la dieta más costosa es la de Greene modificada 8.600 pesos colombianos/ $500 \mathrm{~mL}$ de dieta (US\$1,00 $=\$ 2300$ pesos colombianos), haciéndola un $48 \%$ más costosa que la de Shorey $\mathcal{E}$ Hale modificada y en un $41 \%$ más cara que la dieta
ICRISAT. Es importante mencionar que el agar, en todos los casos, es el ingrediente con mayor participación en los costos. Por esto, trabajos como el de Abbasi et al. (2007), evaluaron con resultados positivos, el almidón de yuca como reemplazo, logrando reducir los costos en más del $50 \%$.

Además, se debe tener en cuenta el número de ingredientes y su disponibilidad en el mercado local y, además, la ejecución de algún proceso, como moler el fríjol, el garbanzo, la soya, o triturar el germen de trigo, lo que incrementa el costo por el aumento en la mano de obra. De allí, no solo es más costosa la dieta Greene, en cuanto al precio de los ingredientes, sino también por la consecución de todos los ingredientes y la adecuación previa del fríjol blanco, harina de soya y la trituración del germen de trigo.

Los costos de producción de 100 larvas de III instar, de 100 pupas y 10.000 huevos, donde se tuvo en cuenta la supervivencia y el consumo de dieta de las larvas hasta este instar, se encuentra en la Tabla 4. La cantidad de dieta que se tomó fue $0,3 \mathrm{~mL} /$ larva, reportada por Murúa et al. (2003). El menor costo, se evidenció con la dieta Shorey $\varepsilon$ Hale modificada, sin embargo, no presenta mayores diferencias con relación a la dieta ICRISAT.

El estado de pupa corresponde a otro parámetro importante para la producción de machos estériles (Alan E Robert, 1996). Para este caso, se tuvo en cuenta la

Tabla 4. Costo en pesos colombianos de la dietas para la producción de 100 larvas instar III, de 100 pupas y de 10.000 huevos de Spodoptera frugiperda.

\begin{tabular}{|c|c|c|c|c|c|c|c|c|c|c|}
\hline & \multicolumn{3}{|c|}{$\%$ de supervivencia } & \multicolumn{3}{|c|}{ \# larvas necesarias para } & \multirow{2}{*}{$\begin{array}{c}\text { \# adultos } \\
\text { Hembra }\end{array}$} & \multicolumn{3}{|c|}{ Costo dieta } \\
\hline & $L$ & $P$ & $\mathrm{H}$ & L & $P$ & $\mathrm{H}$ & & $L$ & $P$ & $\mathrm{H}$ \\
\hline $\mathrm{T} 2$ & 98 & 93 & 83 & 102 & 107 & 13 & 11 & 355 & 1863 & 226 \\
\hline T3 & 96 & 79 & 70 & 104 & 126 & 18 & 12 & 316 & 1912 & 273 \\
\hline T4 & 74 & 21 & 20 & 135 & 476 & 100 & 20 & 696 & 12269 & 2577 \\
\hline
\end{tabular}

En la columna número de larvas, se indica la cantidad de larvas que se requiere criar, para producir 100 larvas instar III, 100 pupas y 10.000 huevos.

El porcentaje de supervivencia, se refiere al acumulado de supervivencia.

$\$ 2.300$ pesos colombianos equivalen aproximadamente a US\$1,00, según el tipo de cambio de enero de 2009. 

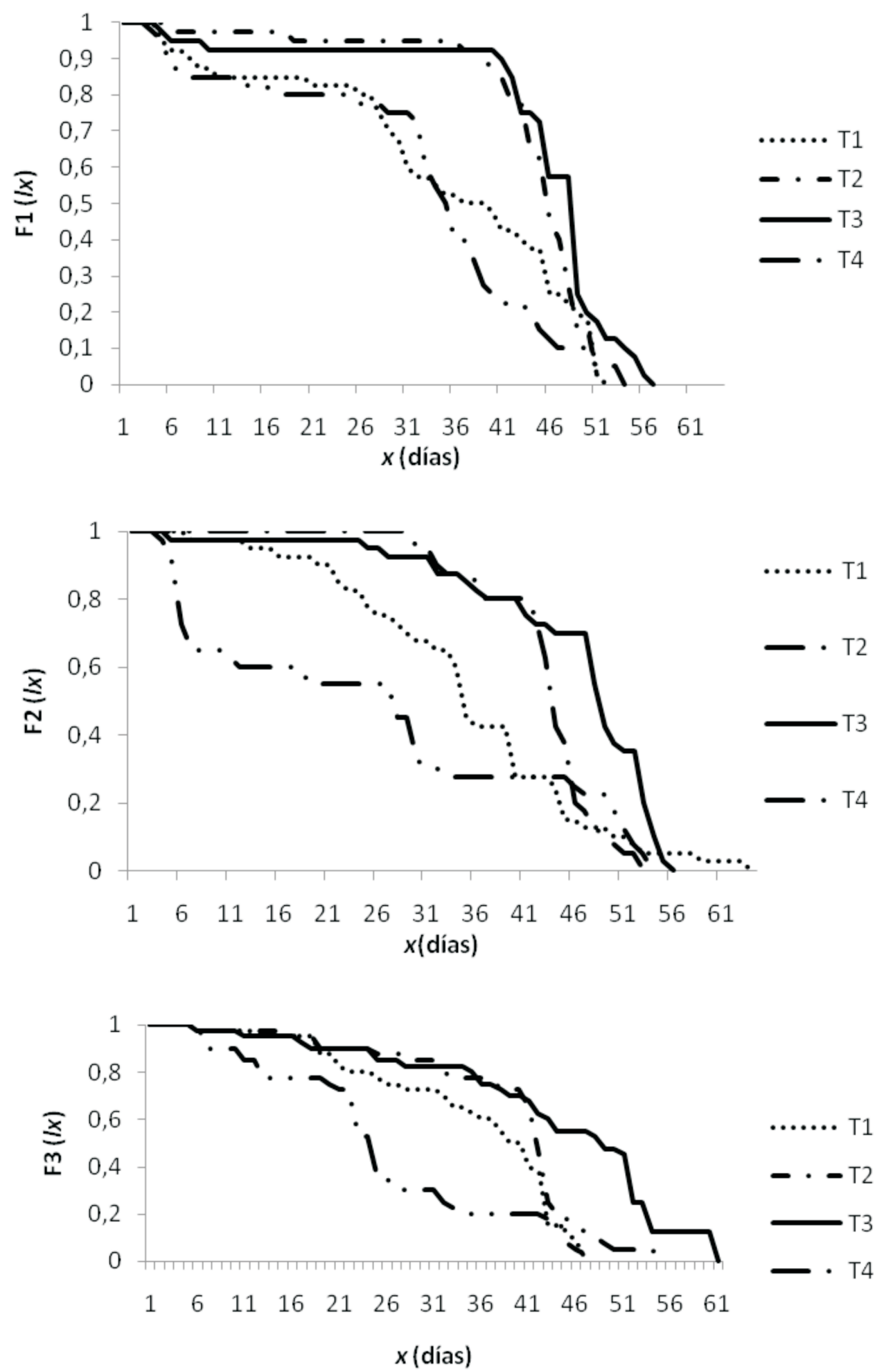

Figura 3. Curvas de supervivencia para Spodoptera frugiperda, en tres dietas artificiales y alimento natural (hojas de maíz), durante tres generaciones consecutivas. T1: Alimento natural (Hojas de Maíz); T2: Dieta ICRISAT; T3: Dieta Shorey \& Hale modificada; T4: Dieta Greene modificada.

F1: primera generación; F2: segunda generación, F3: tercera generación (en laboratorio). 
supervivencia hasta el estado de pupa y el consumo larval, el cual, es de 1,5mL/larva, según Murúa et al. (2003). El menor costo para la producción de 100 pupas, se logró con la dieta ICRISAT, menor en un 2,63\%, respecto a la dieta de Shorey $\mathcal{E}$ Hale y 5,58 veces en relación a la dieta Greene.

Para el cálculo de los costos de las dietas para la producción de 10.000 huevos, se tuvo en cuenta la cantidad de alimento consumido por las larvas, hasta la formación de la pupa, 1,5mL, según Murúa et al. (2003) y la fecundidad de las hembras. En este caso, el menor costo, también se presentó con la dieta ICRISAT, debido a la baja mortalidad observada en esta dieta, principalmente.

\section{CONCLUSIONES}

S. frugiperda, se desarrolló satisfactoriamente sobre las dietas evaluadas; sin embargo, se observaron diferencias a nivel de supervivencia, de duración, de número de instares larvales y de pesos de larva y pupa y de costos. De las dietas evaluadas en ICRISAT Diet 3, el desarrollo de los individuos fue mejor, además, corresponde a la más económica. Para futuros bioensayos, el grupo de investigación empleará, por lo tanto, esta dieta.

Agradecimientos: Al grupo de investigación "Fitosanidad" de la Universidad de Ciencias Aplicadas y Ambientales U.D.C.A y a COLCIENCIAS, por la cofinanciación del presente trabajo. Al doctor José Alonso Álvarez Rodríguez (Q.E.P.D.), por su tutoría, idea inicial y enseñanzas. A Óscar Delgado, Corpoica, La Selva (Rionegro, Antioquia), por su colaboración en el análisis de los datos.

\section{BIBLIOGRAFÍA}

ABBASI, B.; AHMED, K.; KHALIQUE, F.; AYUB, N.; LIU, H.; KAZMI, S.; AFTAB, M. 2007. Rearing the cotton bollworm, Helicoverpa armigera, on a tapiocabased artificial diet. J. Insect Sci. 7:35-42.

ALAN, C.B.; ROBERT, T.S. 1996. El Método de la Liberación de Insectos Estériles y Otras Estrategias de Control Genético. Radcliffe's El texto mundial de MIP. Universidad de Minnesota. Disponible desde Internet en: http://ipmworld.umn.edu/
cancelado/Spchapters/bartlettSp.htm (con acceso el 02/06/2008).

ÁLVAREZ, J.A.; SÁNCHEZ, G. 1983. Variación en el número de instares de Spodoptera frugiperda (J.E. Smith). Rev. Col. de Entomol. 9:43-49.

ÁLVAREZ, J.A. 1991. Reseña histórica y aspectos bioecológicos del gusano cogollero del maíz Spodoptera frugiperda (J. E. Smith). En: Zuluaga, J. L.; Muñoz, G. eds. Memorias Seminario Spodoptera frugiperda en sorgo, maíz y otros cultivos. Socolen-Cis. Calí, Colombia. p.12-16.

BOWLING, C.C. 1967. Rearing of two lepidopterous pests of rice on a common artificial diet. Ann. Ent. Soc. Am. 60(6):1215-1216.

BUSATO, G.R.; GRÜTZMACHER, A.D.; GARCÍA, M.S.; GIOLO, F.P.; ZOTTI, M.J.; MAGALHAES, T.R. 2004. Tabela de vida de fertilidade de populações de Spodoptera frugiperda (J.E. SMITH, 1797) (LEPIDOPTERA: NOCTUIDAE) em folhas de milho e arroz irrigado. Rev. Brás. Agrociencia. 10(4):449-455.

BUSATO, G.R.; GARCÍA, M.S.; LOECK, A.E.; ZART, M.; NUNES, A.M.; BERNARDI, O.; ANDERSSON, F.S. 2006. Adequação de uma dieta artificial para os biótipos "Milho" e "Arroz" de Spodoptera frugiperda (Lepidoptera: Noctuidae). Bragantia, Campinas. 65(2):317-323.

BUSTILLO, A. E. 1979. La nutrición en insectos. Sociedad Colombiana de Entomología. Medellín. Boletín de Divulgación. Número 2. 43p.

CRUZ, I.; WAQUIL, J.M.; VIANA, P. A. 1995. Pragas: diagnóstico e controle. Arquivo do Agrônomo. 2:10-14.

GALLEGO, F.L. 1946. Plagas del maíz Laphygma frugiperda S. and A. Universidad Nacional de Colombia eds (Medellín). Estudio Fundamental No. 5. 60p.

GARCÍA, M.S.; BUSATO, G.R.; GIOLO, F.P.; MANZONI, C; BERNARDI, O.C.; ZART, M.; NUNES, A.M. 2006. Tabela de vida de fertilidade de Helicoverpa 
zea (Boddie, 1850) (Lepidoptera: Noctuidae) em duas dietas artificiais. Rev. Bras. Agrociencia (Pelotas). 12(1):51-55.

GREENE, G.L.; LEPLA, N.C.; DICKERSON, W.A. 1976. Velvetbean caterpillar: a rearing procedure and artificial medium. J. Econ. Entom. Lanham. 69(4):488-497

GRÜTZMACHER, A.D.; MARTINS, J.F.; CUNHA, U.S. 2000. Da Insetos-pragas das culturas do milho e sorgo no agroecossistema de várzea. In: PARFITT, J.M.B. Produção de milho e sorgo em várzea. Pelotas: Embrapa Clima Temperado. p.87-102.

KOGAN, M. 1998. Integrated pest management: historical perspective and contemporary development. Ann. Rev. Entomol. 43:243-270.

MARÇON, P.C.R.G.; SIEGFRIED, B.D.; SPENCER, T.; HUTSCHISON, W.D. 2000. Development of diagnostic concentrations for monitoring Bacillus thuringiensis resistance in european corn borer (Lepidoptera: Cambrinae). J. Econ. Entomol. 93(3):925-930.

MURÚA, M.G.; VIRLA, E.G.; DEFAGÓ, V. 2003. Evaluación de cuatro dietas para la cría de Spodoptera frugiperda (Lepidoptera: Noctuidae) destinada a mantener poblaciones experimentales de himenópteros parasitoides. Bol. San. Veg. Plagas (Argentina). 29:43-51.

PANTOJA, A.; SMITH, C.M.; ROBINSON, J.F. 1987. Development of fall armyworm Spodoptera frugiperda (J. E. Smith) (Lepidoptera: Noctuidae), strains from Louisiana and Puerto Rico. Envion. Entomol. 16(1):116-119.

PARRA, J.R. 2001. Técnicas de criação de insetos para programas de controle biológico. Piracicaba: ESALQ 134p.

RAVINOVICH, J.E. 1980. Introducción a la ecología de poblaciones animales. CECSA. Comp. Edit. Continental. México. p.105-135.

SARMENTO, R.A.; AGUIAR, R.W.; AGUIAR, R.A.; VIEIRA, S.M.; OLIVEIRA, H.G.; HOLTZ, A.M. 2002. Revisão da Biologia ocorrência e controle de Spodoptera frugiperda (LEPIDOPTERA, NOCTUIDAE) em milho no Brasil. Bioscience J. 18(2):41-48.

SHOREY, H.H.; HALE, R.L. 1965. Mass-Rearing of the larvae of nine Noctuid species on simple artificial medium. J. Econ. Entomol. 58:522-524.

SMITH, B.R. 1921. Observations on the fall armyworm (Laphygma frugiperda Smith \& Abbot) and some control experiments. J. Econ. Entomol. 14(4):300308.

SOUZA M.L., A.; ÁVILA J., C.; PARRA R.P., J. 2001. Consumo e utilização de alimento por Diatraea saccharalis (Fabr.) (Lepidoptera:Pyralidae), Heliothis virescens (Fabr.) e Spodoptera frugiperda (J.E.Smith) (Lepidóptera:Noctuidae) em duas temperaturas. Neotropical Entomology 30(1):1117.

VÉLEZ, R.A. 1985. Notas sinópticas de entomología económica colombiana. Universidad Nacional de Colombia. Medellín. p.145-149.

VILLACORTA, A.; COBO DE MARTÍNEZ, L.S. 1978. Efecto del modo de preparación de la dieta sobre el crecimiento y desarrollo de Spodoptera frugiperda. ICA (Colombia). p.96-103.

ZENNER DE POLANÍA, I.; ÁLVAREZ, J.A.; ARÉVALO, H.M.; MEJÍA R.C.; BAYONA, M.R. 2008. Susceptibilidad de cuatro nóctuidos plaga (Lepidoptera) al gene Cry1Ac del Bacillus thuringiensis incorporado al algodonero. Rev. Col. Entomol. 34(1):41-50.

ZENNER DE POLANÍA, I.; ARÉVALO, H.M.; MEJÍA C., R. 2007. El gusano cogollero del maíz Spodoptera frugiperda (J.E. Smith) (Lepidoptera: Noctuidae) y algunas plantas transgénicas. Rev. Col. Cienc. Hortícolas. 1(1):103-113.

Recibido: Enero 5 de 2009

Aceptado: Abril 14 de 2009 